\title{
The AQARA Principle: Proposing Standard Requirements for Radionuclide-Based Images in Medical Journals
}

\author{
Wolfgang A. Weber ${ }^{1}$, Frank M. Bengel ${ }^{2}$, and Ronald G. Blasberg ${ }^{3}$ \\ ${ }^{1}$ Technical University of Munich, Munich, Germany; ${ }^{2}$ Hannover Medical School, Hannover, Germany; and ${ }^{3}$ Memorial Sloan \\ Kettering Cancer Center, New York, New York
}

A of radioactive decays in the body of the studied subject. The number of radioactive decays per unit of time is directly proportional to the concentration of the injected radioisotope. This simple quantitative relationship between the imaging signal and the investigated biologic process is a major strength of nuclear imaging. Of course, the quantitative relationship can be confounded by metabolism of the radiopharmaceutical, as well as by attenuation and scatter of the emitted photons. Nevertheless, nuclear images often provide important quantitative information about biologic mechanisms. This is especially true of PET scans, which are now routinely reconstructed as maps of activity concentrations $\left(\mathrm{Bq} / \mathrm{cm}^{3}\right)$ or normalized activity concentrations, such as SUVs. The quantitative accuracy of PET data is continuously improving because of progress in PET detection systems and reconstruction algorithms. In parallel, attenuation- and scatter-corrected SPECT has also made significant progress.

Nuclear images published in scientific journals should therefore present the reader with information that is "as quantitative as reasonably achievable" (AQARA). We believe that a simple but important step in this direction is to always display nuclear images with an intensity-scale bar that illustrates how the measured signals were converted to color or gray-scale values.

The intensity-scale bar reflects the contrast in the images and greatly facilitates comparison between images from different studies. Furthermore, the scale bar allows the reader to immediately assess the strength of the measured imaging signal and its relationship to background activity. The scale bar also shows the extent of contrast and whether it was increased or decreased by a nonlinear scale or background subtraction was used. Nonlinear scaling and background subtraction may improve the visualization of certain image features but their excessive use can be misleading and may not be easily detected by the reader if no scale bar is shown along with the image.

For CT images, there are well-standardized window settings for display of lung, soft tissue, and bone. It is more difficult to standardize the display of nuclear images because of the often higher dynamic range of the image information and the widely different activity distributions of the various radiotracers. Furthermore, CT images

Received Dec. 4, 2019; accepted Dec. 4, 2019.

For correspondence or reprints contact: Wolfgang A. Weber, Technical University of Munich, Ismaningerstrasse 22, 81675 Munich, Germany.

Email: w.weber@tum.de

COPYRIGHT @ 2020 by the Society of Nuclear Medicine and Molecular Imaging. DOI: 10.2967/jnumed.119.240523 are almost universally displayed using a white-on-black color scale. In contrast, a large variety of gray and color scales are in use for display of different types of nuclear images (mostly for historic reasons). It is now almost impossible to change these traditions and use the same color scale for all nuclear medicine studies. However, a scale bar can make it much easier for the reader to correctly interpret the images displayed with these different color scales and window settings.

To best convey the quantitative information in nuclear images and to standardize their display, we therefore propose 5 key requirements for a standardized radionuclide imaging figure:

1. If feasible, the intensity-scale bar should be in absolute units, for example, $\mathrm{Bq} / \mathrm{mL}$ or SUVs. This is the best way to ensure standardized display of images, independent of the specific distribution of radioactivity in an individual scan. Absolute scaling is almost always feasible for PET images and is becoming more common for SPECT images as well. If parametric images showing biologically relevant parameters (such as rate constants, metabolic rates, volumes of distribution, or binding potentials) are available, they should generally be preferred to images of physical parameters such as activity concentrations. The unit used for the scale bar should always be displayed within the image, such as at one side of the scale bar.

2. When no absolute scaling of images is feasible or reasonable (e.g., in myocardial rest/stress perfusion SPECT images), a relative-scale bar should be used. Images may then be scaled to the maximum activity in the displayed image or the investigated organ. The unit of the scale bar would then be percentage of maximum, and the top and bottom values should be identified accordingly. When several images of a tracer are shown within a single published article, they should preferably be displayed with the same upper and lower window settings to facilitate comparisons.

3. Setting pixels with a value below a certain threshold to a background color (background subtraction) should generally be avoided. If necessary or useful for a better display of a specific image feature, the background subtraction should be clearly indicated in the scale bar. The bottom of the scale bar should still be labeled as " 0 ," and the fraction of the scale bar below the background value should be displayed in the background color to indicate the degree of background subtraction.

4. Overlaying of PET and SPECT images on top of anatomically coregistered CT and MR images (image fusion) is helpful to display the exact location of findings on PET and SPECT 
TABLE 1

AQARA Requirements for Radionuclide-Based Images in Medical Journals

\begin{tabular}{|c|c|}
\hline Feature & Specific requirement \\
\hline \multirow[t]{6}{*}{ Intensity-scale bar } & Show for each figure \\
\hline & Indicate unit and upper and lower window settings \\
\hline & Prefer absolute units: \\
\hline & Use biologic parameters if available (e.g., rate constants, metabolic rates, binding potential) \\
\hline & If not, use activity concentration (e.g., SUV, Bq/mL, \%IA/cm³) \\
\hline & Minimally, include relative units (e.g., percentage of maximum) \\
\hline \multirow[t]{4}{*}{ Background subtraction } & Avoid \\
\hline & If unavoidable, clearly indicate in scale bar: \\
\hline & Keep bottom of scale bar at "0" \\
\hline & Set subtracted fraction of scale bar to background color \\
\hline \multirow[t]{4}{*}{ Image display } & Use same window settings for all figures \\
\hline & $\begin{array}{l}\text { Accompany fusion images (SPECT/CT, PET/CT, PET/MR) by corresponding stand-alone } \\
\text { SPECT or PET images }\end{array}$ \\
\hline & Limit cropping \\
\hline & If cropping is applied, show corresponding uncropped, full-field-of-view image \\
\hline
\end{tabular}

images. However, during the coregistration process, some information from the PET and SPECT images can be lost. For example, low-grade tracer uptake in areas that are dark on CT and MR images may become difficult or impossible to evaluate. Therefore, figures should show not only fused images but also the individual PET and SPECT images. Preferably, these images should be displayed in a black-on-white intensity scale.

5. Cropping of images should be limited, except for wellestablished imaging procedures such as myocardial perfusion. If cropping is necessary to show small details, an uncropped image illustrating the tracer distribution in the entire field of view should additionally be shown - as a supplementary figure, for example. This proposed requirement is especially important for reports on new radiopharmaceuticals.

Table 1 summarizes the key points and may serve as a checklist for preparation of figures. We believe that these proposed requirements are easy to follow and will help readers to correctly interpret nuclear images. As of the publication date of this editorial, The Journal of Nuclear Medicine will therefore begin applying these requirements. Specifically, intensity-scale bars will become mandatory for all PET, SPECT, and planar nuclear images submitted for publication in The Journal of Nuclear Medicine. 